\title{
Cosmic magnetic field observations with next generation instrumentation
}

\author{
Rainer Beck \\ Max-Planck-Institut für Radioastronomie, Auf dem Hügel 69, 53121 Bonn, Germany \\ email: rbeck@mpifr-bonn.mpg.de
}

\begin{abstract}
The origin of magnetic fields in the Universe is an open problem in astrophysics and fundamental physics. Forthcoming radio telescopes will open a new era in studying cosmic magnetic fields. Low-frequency radio waves will reveal the structure of weak magnetic fields in the outer regions and halos of galaxies and in intracluster media. At higher frequencies, the EVLA and the SKA will map the structure of magnetic fields in galaxies in unprecedented detail. All-sky surveys of Faraday rotation measures (RM) towards a huge number of polarized background sources with the SKA and its pathfinders will allow us to model the structure and strength of the regular magnetic fields in the Milky Way, the interstellar medium of galaxies, in galaxy clusters and the intergalactic medium.
\end{abstract}

Keywords. Techniques: polarimetric, galaxies: clusters, galaxies: magnetic fields

\section{Origin of cosmic magnetic fields}

The origin of the first magnetic fields in the Universe is still a mystery (Widrow 2002). A large-scale primordial field is hard to maintain in a young galaxy because the galaxy rotates differentially, so that field lines strongly wind up and reconnect during galaxy evolution. Turbulent "seed" fields in young galaxies can originate e.g. from the Weibel instability in shocks during cosmological structure formation (Lazar et al. 2009), or can be injected by the first stars or jets generated by the first black holes (Rees 2005). In any case a mechanism to sustain and organize the magnetic field is required. The most promising mechanism is the dynamo (Beck et al. 1996) which transfers mechanical energy into magnetic energy. In young galaxies a small-scale dynamo with turbulence driven by star formation (Brandenburg \& Subramanian 2005) probably amplified the seed fields from the protogalactic phase to the energy density level of turbulence within less than $10^{9} \mathrm{yr}$, followed by a large-scale dynamo generating regular fields (2009).

Magnetic fields in galaxy clusters are probably seeded by AGNs and amplified in the intracluster medium by a turbulent dynamo (Ryu et al. 2008), giving rise to diffuse radio halos. Cluster mergers may compress the field and generate "radio relics" which are highly polarized and best observable at low frequencies.

\section{Tools to measure interstellar magnetic fields}

Most of what we know about galactic and intergalactic magnetic fields comes through the detection of radio waves. Synchrotron emission is a measure of the strength of the total magnetic field component in the sky plane. Linearly polarized synchrotron emission emerges from ordered fields, and $B$-vectors indicate the field orientation. The orientation of the polarization vectors is changed in a magnetized thermal plasma by Faraday rotation. Faraday rotation measures (RM) from multi-wavelength observations yield the strength and direction of the regular field component along the line of sight. Zeeman splitting of radio spectral lines also measures the regular field along the line of sight.

\section{Prospects with future telescopes}

Future radio telescopes will greatly widen the range of observable magnetic phenomena. To resolve the detailed structure of the ISM and halo fields, high-resolution, deep polarization 
observations at high frequencies, where Faraday effects are small, require a major increase in sensitivity for continuum observations which will be achieved by the Extended Very Large Array (EVLA) and the planned Square Kilometre Array (SKA). Polarized emission is an excellent tracer of interactions between galaxies and of cluster mergers. The SKA will also allow to measure the Zeeman effect in weak magnetic fields in the Milky Way and in nearby galaxies.

Low-frequency radio telescopes like the Low Frequency Array (LOFAR, under construction) and the low-frequency SKA array will be suitable instruments to search for synchrotron radiation from low-energy cosmic-ray electrons which suffer only moderately from energy losses, allowing to trace magnetic fields in the outer regions and halos of galaxies, in the Milky Way and in galaxy clusters (Beck 2009).

Radio spectro-polarimetric observations in many narrow frequency channels allows application of $R M$ Synthesis (Brentjens \& de Bruyn 2005). If the emitting medium has a relatively simple structure, Faraday tomography will become possible. This method is going to revolutionize radio polarization observations.

Polarized pulsars and extragalactic sources can be used to observe a grid of RM values to measure the magnetic field structure of intervening objects. A sufficiently high number density of sources requires large sensitivity and/or high survey speed. The POSSUM all-sky survey at $1.4 \mathrm{GHz}$ with the planned Australia SKA Pathfinder (ASKAP) telescope will measure about 80 RM per square degree. The SKA will be able to detect several $1000 \mathrm{RM}$ per square degree. The SKA Magnetism Key Science Project plans an all-sky RM grid which will contain about $10^{4}$ RM values from pulsars with a mean spacing of $\simeq 30^{\prime}$ and about $10^{8} \mathrm{RM}$ from compact polarized extragalactic sources at a mean spacing of just $\simeq 1.5^{\prime}$ (Gaensler et al. 2004). This survey will be used to model the structure and strength of the magnetic fields in the Milky Way, in intervening galaxies, and in the intergalactic medium. The distance range of this method is much larger than by direct imaging of the polarized emission from the intervening galaxy. Looking back into time, the future telescopes will also shed light on the origin and evolution of cosmic magnetic fields in galaxies (Kronberg et al. 2008) and in clusters (Krause et al. 2009). The detection of RM from the intergalactic medium would allow us to probe the existence of magnetic fields in such rarified regions, measure their intensity, and investigate their origin and their relation to the structure formation in the early Universe.

\section{References}

Arshakian, T. G., Beck, R., Krause, M., \& Sokoloff, D. 2009, A\&A A, 494, 21

Beck, R. 2009, Rev. Mex. AyA 36, 1

Beck, R., Brandenburg, A., Moss, D., Shukurov, A., \& Sokoloff, D. 1996, ARAA 34, 155

Brandenburg, A. \& Subramanian, K. 2005, Phys. Rep. 417, 1

Brentjens, M. A. \& de Bruyn, A. G. 2005, A\&\& 441, 1217

Gaensler, B. M., Beck, R., \& Feretti, L. 2004, New Astr. Rev. 48, 1003

Krause, M., Alexander, P., Bolton, R., et al. 2009, MNRAS, in press

Kronberg, P. P., Bernet, M. L., Miniati, F., et al. 2008, ApJ 676, 70

Lazar, M., Schlickeiser, R., Wielebinski, R., \& Poedts, S. 2009, ApJ 693, 1133

Rees, M. J. 2005, in: R. Wielebinski, \& R. Beck (eds.), Cosmic Magnetic Fields (Berlin: Springer), p. 1

Ryu, D., Kang, H., Cho, J., \& Das, S. 2008, Science 320, 909

Widrow, L. M. 2002, Rev. Mod. Phys. 74, 775 